\title{
An Overview of a New Sensor Calibration Platform
}

\section{P. Clausen ${ }^{1}$, J. Skaloud ${ }^{1}$, R. Molinari ${ }^{2}$,}

\section{J. Balamuta ${ }^{3}$, W. Yang ${ }^{3}$, J. Lee $^{3}$, S. Guerrier ${ }^{3}$}

\begin{abstract}
${ }^{1}$ Geodetic Engineering Laboratory, Ecole Polytechnique Fédérale de Lausanne, Switzerland ${ }^{2}$ Department of Statistics and Applied Probability, University of California, Santa Barbara, USA

${ }^{3}$ Department of Statistics, University of Illinois, Urbana-Champaign, USA
\end{abstract}

\section{Goal: Stochastic Modeling of (Inertial) Sensors}

Inertial sensors have been employed in different types of applications in miniature embedded devices such as phones, watches, and small unmanned aerial vehicles. The stochastic structure of the error signal coming from these sensors is complex and needs to be determined for optimal fusion with other devices (e.g. GPS). The recently-proposed approach, Generalized Method of Wavelet Moments (GMWM) using the wavelet variance (WV), overcomes these limitations. The presented software platform can be used for stochastic calibration (i.e. parameter determination) of IMUs or other types of sensors. The software is Open Source and is developed within the broadly used statistical framework $\mathbf{R}$ using $\mathbf{C + +}$ language.

\section{What is behind GMWM?}

GMWM finds a solution to the minimization problem, by looking for the proper parameters that match the 'empirical $W V^{\prime}$ ' and the 'model-implied WV'. Possible processes are: Quantization Noise (QN), White Noise (WN), Gauss Markov (GM), Random Walk (RW) and Drift (DR).

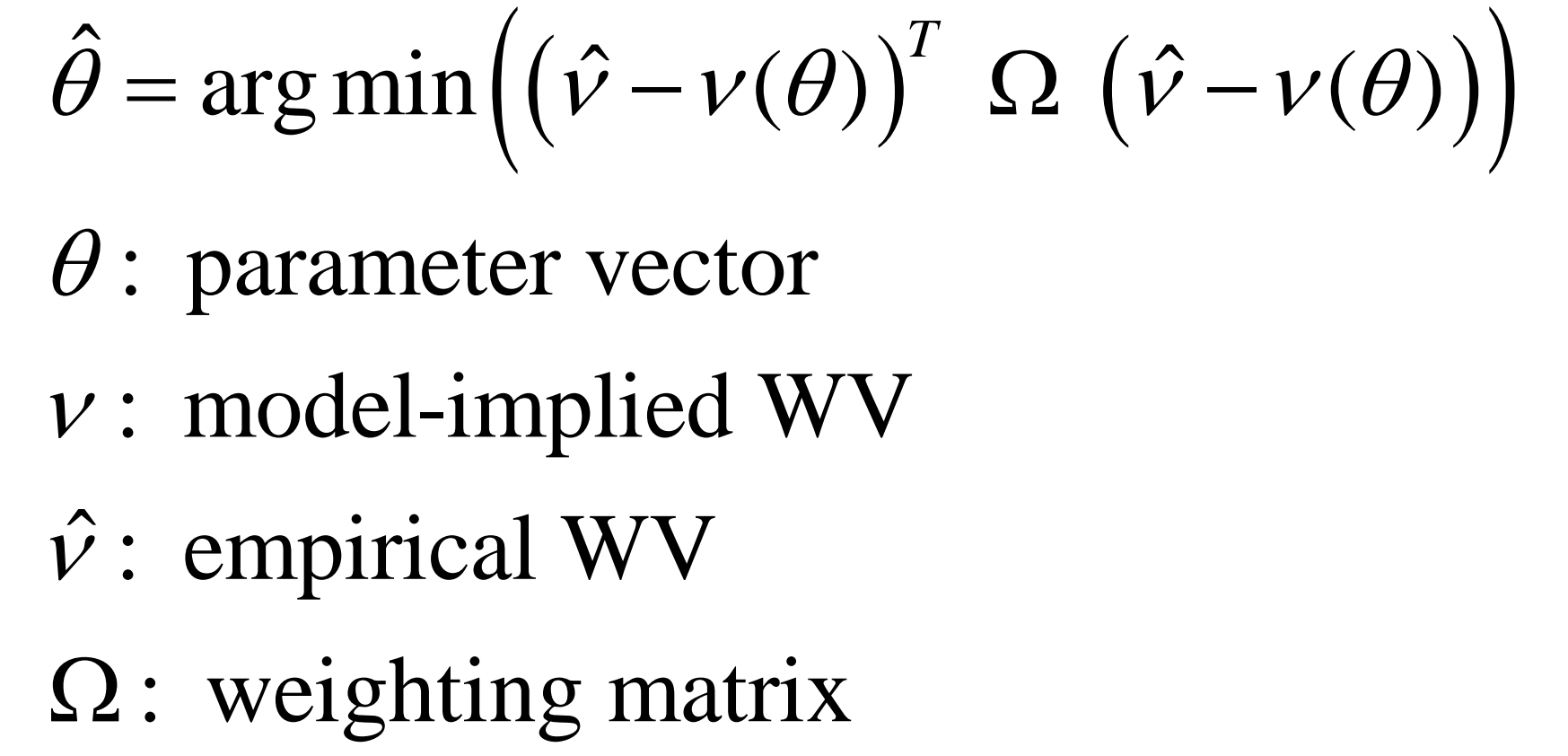

\section{Calibration example}

Calculation of the empirical WV from several hours

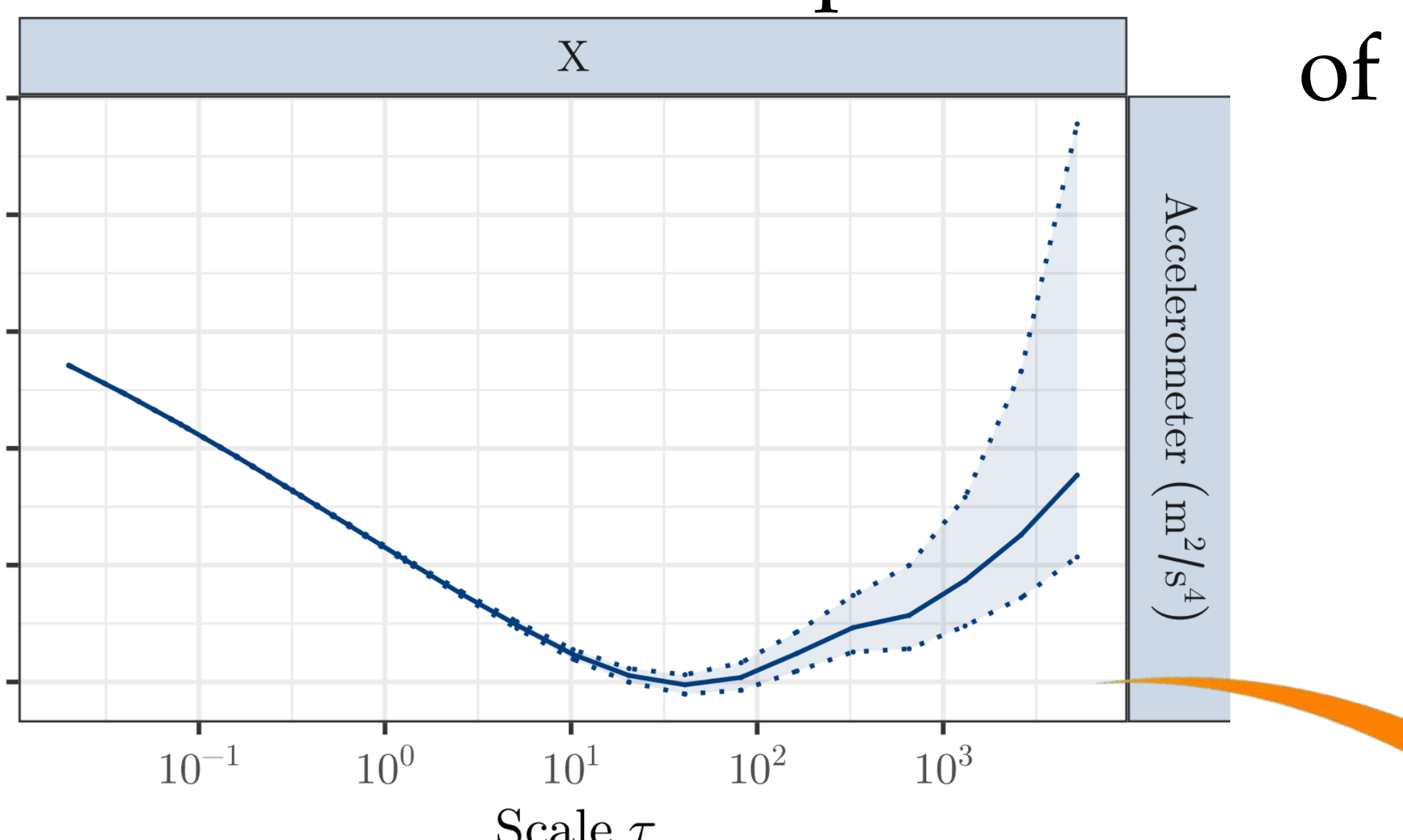
of static IMU data.

Modeling with WN, RW and 1XGM.

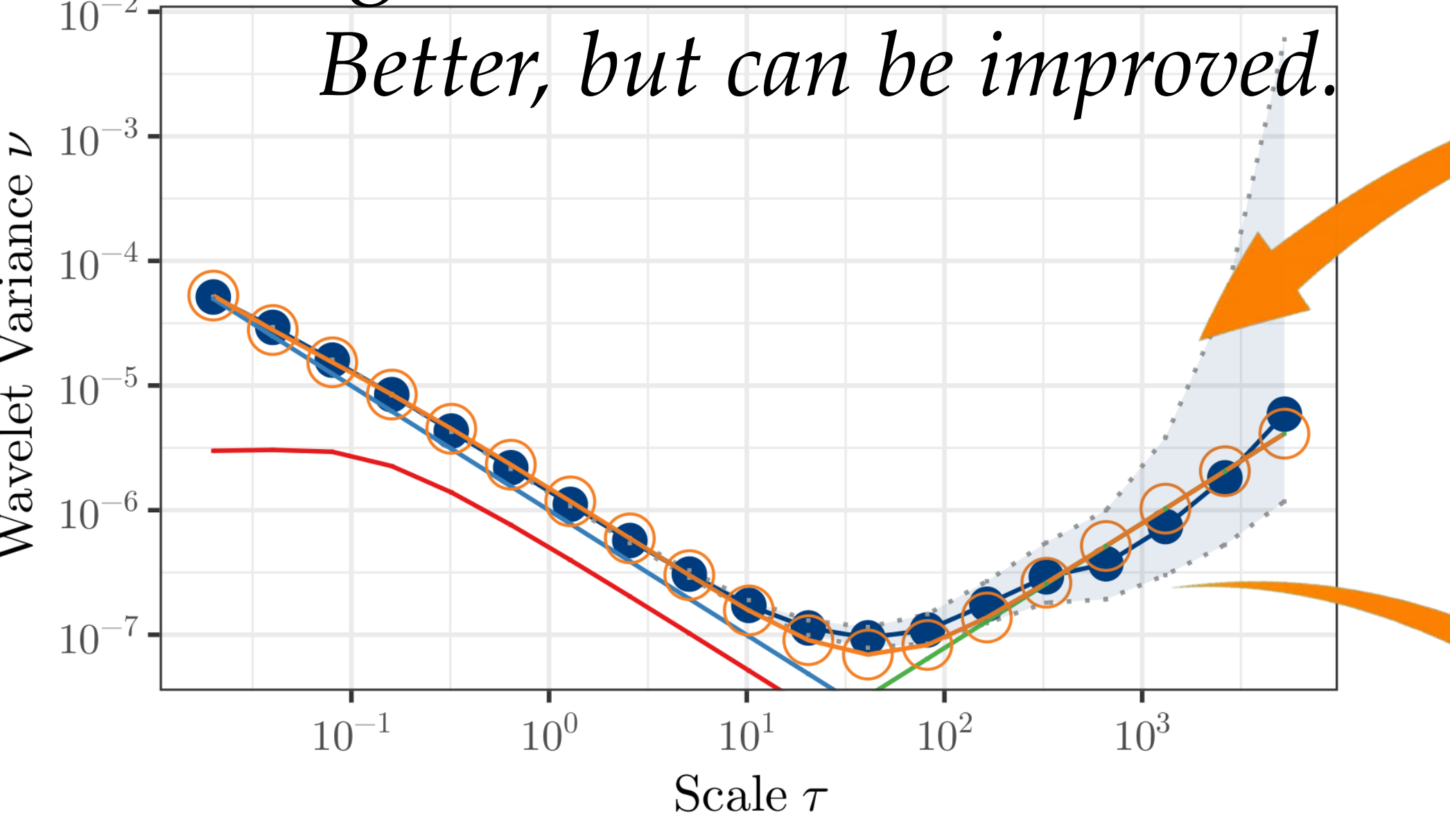

Modeling a $W N, R W, 2 x G M$ and $D R$.

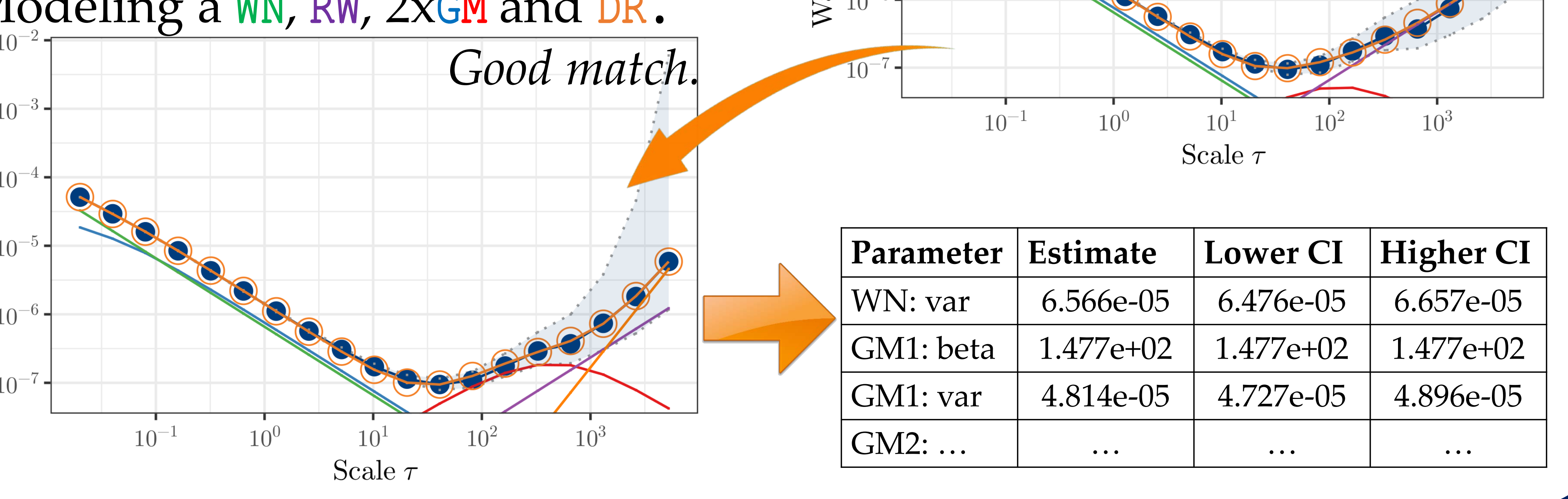

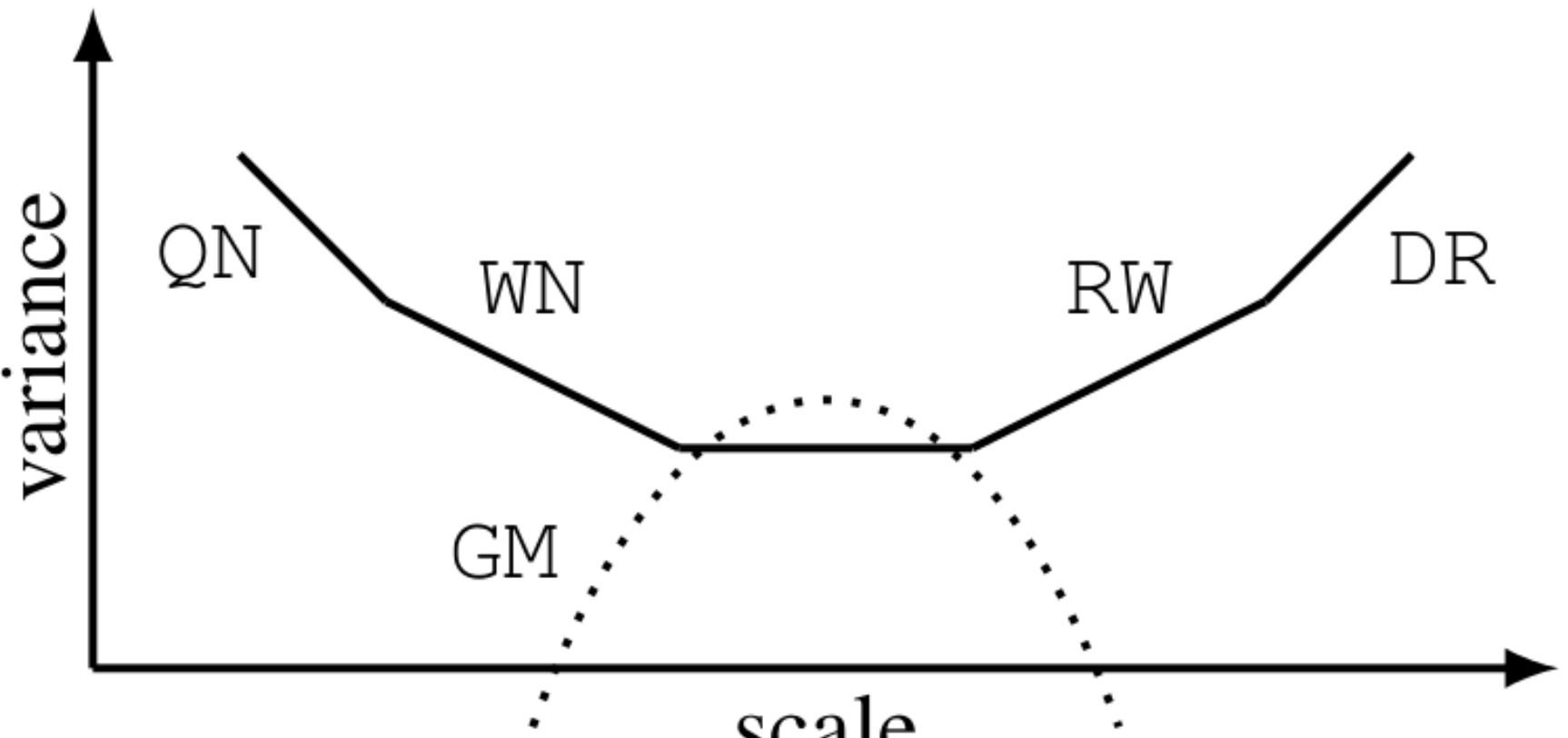

Modeling with WN and RW.

Empirical WV: blue dots. Modelimplied WV: orange circles. But it does not fit quit well

\section{Application}

Each sensor has its own noise characteristics. High grade IMUs (blue) have a precise and easy model, whereas low-cost IMUs (orange) show a more complicated error structure.

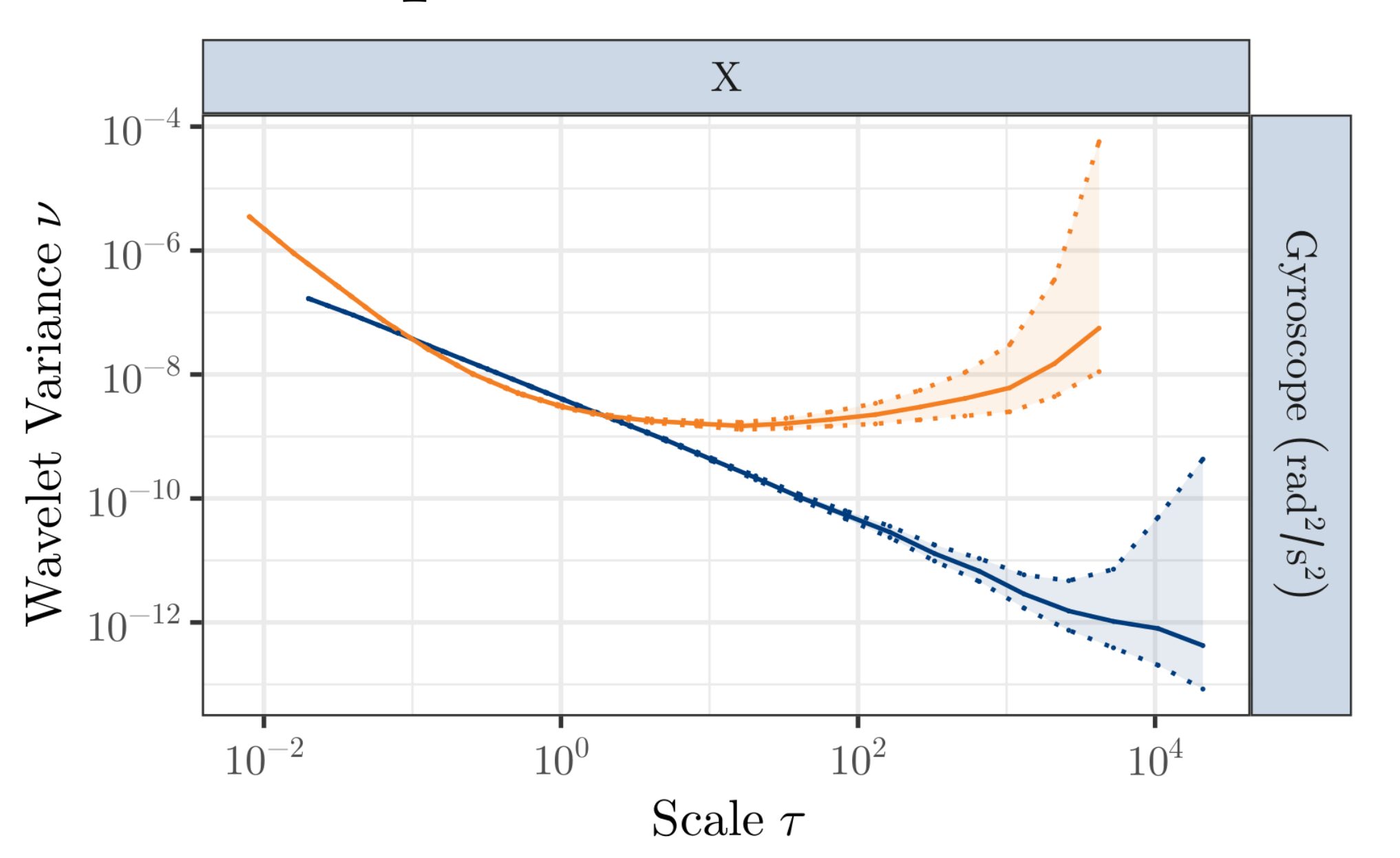

Precise knowledge of IMU sensor stochastics improves the navigation solution. The GMWM can provide these parameters.
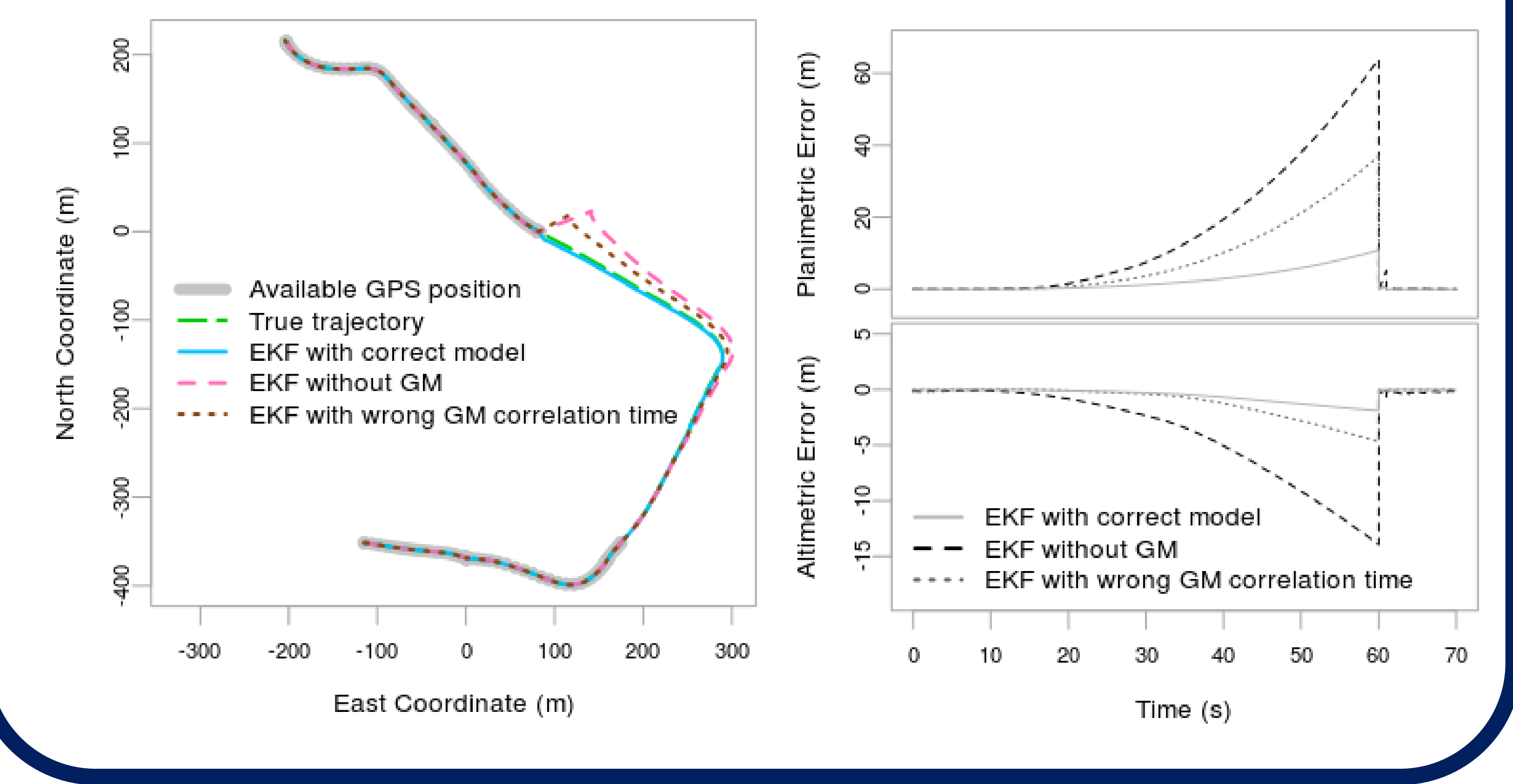

\section{Conclusion}

Modeling with WN, RW and 2xGM. Good, can I push it further?

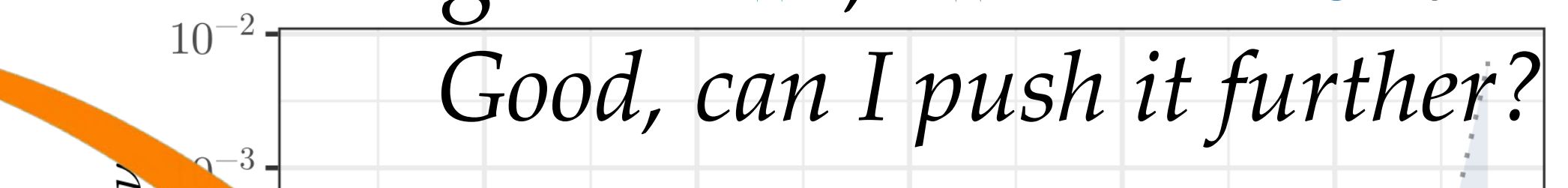

